\title{
Gold Nanoparticle Doped PVDF Nanofiber Preparation of Concurrently Harvesting Light and Mechanical Energy
}

\author{
Binoy Bera ${ }^{1}$, Madhumita Das Sarkar ${ }^{2}$ \\ ${ }^{1,2}$ Department of computer science and engineering, West Bengal University of Technology, Kolkata - 700064, \\ India
}

\begin{abstract}
PVDF (polyvinylidene fluoride) is a very well known material for converting mechanical energy into electrical energy and gold nanoparticle are versatile material for a broad range of applications with well characterized electronic and physical properties. In this work, we developed gold nanoparticle doped PVDF nanofiber based nanogenerators which are concurrently harvest light and mechanical energy into electrical energy. Here nanofiber has been made by electrospinning process. Characterization of fiber has been done by FE - SEM instrument, UV - VIS spectroscopy instrument and FTIR instrument. Beside this, characterization of film $\left(\right.$ Casted at $120^{\circ} \mathrm{c}$ ) has also been done. For nanogenerator preparation ITO coated polyethylene terephthalate (PET) and silver fabric were used as electrodes. Finally polydimethylsiloxane (PDMS) were used for coating these nanogenerators. Electrical output data have taken in different combination of light and mechanical energy and calculated the damping ratio of these nanogenerators (before PDMS coating) in cantilever geometry. In future $G O, R G O$ or Semiconductor quantum dots (QDS) can be incorporated into this nanofiber based nanogenerator for improvement of better optical, electrical and/or electronic properties.
\end{abstract}

Keywords: PVDF, Nanogenerator, Energy harvesting, Electrospinning process, Gold nanoparticle.

\section{Introduction}

Nanogenerators (NGs) based on the piezoelectric ${ }^{[1,2,3]}$, triboelectric ${ }^{[4]}$ or pyroelectric ${ }^{[5]}$ effect, which can convert trace energy in the environment into electric energy, have performed as an efficient energy source for nanodevices and nanosystems. Self-powered nanosystems have been proved viable in self-powered $\mathrm{pH}$ sensors ${ }^{[6]}$, UV sensors ${ }^{[7]}$, self-charging power cells ${ }^{[8]}$, small liquid crystal displays ${ }^{[9]}$, commercial laser diodes ${ }^{[10]}$, etc. Piezoelectric nanogenerators with the ability of converting ambient mechanical energy to electric power can be an interesting candidate for energy harvesting applications. These fabricated nanogenerators by nanomaterials have attracted lots of attentions since the first report on utilizing piezoelectric zinc oxide nanowires for mechanical energy harvesting in $2006{ }^{[3]}$. More and more piezoelectric nanomaterials have been researched in mechanical energy scavenging such as $\mathrm{ZnO}^{[11,12,13]}, \mathrm{ZnS}^{[14]}, \mathrm{GaN}^{[15]}, \mathrm{PZT}^{[16,17]}$ and BaTiO3 ${ }^{[18]}$. Compared with those inorganic nanomaterials in realistic applications, piezoelectric polymers are promising alternatives for energy harvesting devices. These materials combine structural flexibility, chemical stability with ease of processing ${ }^{[19]}$ and simplicity in device designing. Especially, polyvinylidene fluoride (PVDF) has a relatively high piezoelectric property owing to its polar crystalline structure, consisting of at least five different crystalline forms (namely $\alpha, \beta, \gamma, \delta$ and $\varepsilon)^{[20,21]}$, among which $\beta$ phase is primary for its piezoelectric activity. On the other hand, electrospinning is an effective way on $\beta$ phase formation which combines electrical poling and uniaxial stretching in one step ${ }^{[22]}$.

Gold nanoparticles ${ }^{[23]}$ interaction with light is strongly dictated by their environment, size and physical dimensions. Oscillating electric fields of a light ray propagating near a colloidal nanoparticle interact with the free electrons causing a concerted oscillation of electron charge that is in resonance with the frequency of visible light. These resonant oscillations are known as surface plasmons. For small ( $\sim 30 \mathrm{~nm})$ monodisperse gold nanoparticles the surface plasmon resonance phenomona causes an absorption of light in the blue-green portion of the spectrum $(\sim 450 \mathrm{~nm})$ while red light $(\sim 700 \mathrm{~nm})$ is reflected, yielding a rich red color.

In this work gold nanoparticle doped PVDF nanofiber (prepared by electrospinning process) based nanogenerator has been demonstrated for harvesting light and mechanical energy into electrical energy. Characterization of electrospinning fiber has been done by FE - SEM, UV - VIS and FTIR instrument. Then this nanogenerator are coated by Polydimethylsiloxane(PDMS). Finally the output voltage of this nanogenerator have been taken in different combination of light and mechanical energy. The damping ratio in cantilever geometry (before PDMS coating) is also measured. 


\subsection{Materials}

\section{Experimental Procedure}

Poly (vinylidene fluoride) (PVDF) pellets ( $\overline{\mathrm{M}} \mathrm{w} \approx 275000$, Sigma-Aldrich, USA), N, Ndimethylformamide (DMF), acetone (Merck Chemical, India), chloroauric acid ((Sigma-Aldrich, Germany), Indium tin oxide coated PET(Sigma-Aldrich, Germany).

\subsection{Gold Nanoparticle preparation}

For the preparation of gold nanoparticle , $10 \mu \mathrm{M}$ of HAuCl4.3H2O (Sigma-Aldrich, Germany) were added to $12 \mathrm{wt} \%$ (w/v) PVDF-DMF (Sigma-Aldrich, Germany) solutions which were afterwards vigorously stirred at $32^{\circ} \mathrm{C}$ for $6 \mathrm{~h}$.

\subsection{Electrospinning sample preparation}

For the preparation of electrospinning sample, first $300 \mu 1,500 \mu 1$ and $700 \mu 1$ of previously prepared gold nanoparticle solutions has been added in $12 \mathrm{wt} \%(\mathrm{w} / \mathrm{v})$ PVDF - DMF solution respectively. Then added 4 $\mathrm{ml}$ of acetone with it and stirred (30 minutes) by magnetic stirrer for final solution $(10 \mathrm{ml})$ of electrospinning process. Then loaded this samples in syringe $(20 \mathrm{ml})$ for electrospinning process. We placed our ITO coated PET onto aluminium foil for collecting the electrospinning fiber.

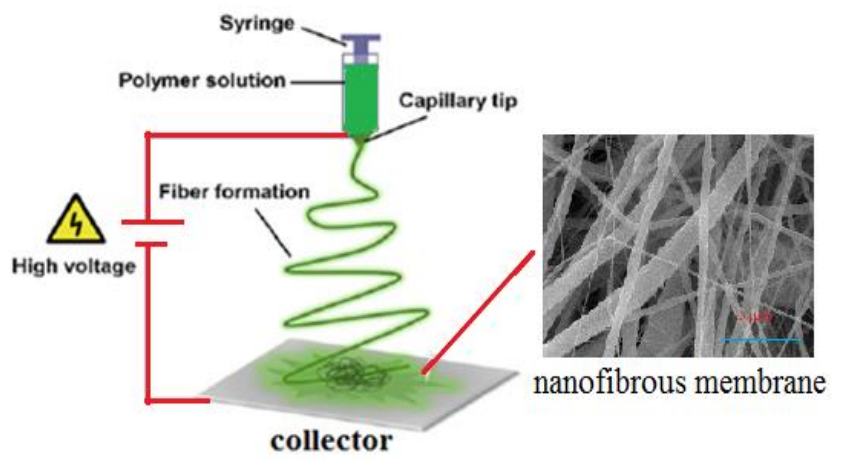

Fig.1. Schematic illustration of the basic setup for electrospinning. The inset shows a typical SEM image of the PVDF nanofibrous membranes deposited on the collector.

\subsection{Nanogenerator preparation}

For the nanogenerator preparation, silver fabrics and ITO coated PET is used as electrodes. After that this nanogenerators has been coated with polydimethylsiloxane(PDMS).HerePVDF.300AuPVDF, PVDF.500AuPVDF, PVDF.700AuPVDF nanogenerator means 300 $\mu 1,500 \mu 1,700 \mu 1$ of AuPVDF solutions are concurrently mixed with $12 \mathrm{wt} \%$ PVDF - DMF solution respectively. In addition we have also casted the solution of electrospinning samples(before the addition of acetone) at $120^{\circ} \mathrm{c}$ for 4 hours for the film characterization. Next measured the the electrical output of these nanogenerators in presence of light and mechanical energy. Before the coating of PDMS the output response in cantilever geometry have been taken. Capacitor $(4.7 \mu \mathrm{F})$ charging voltage of PDMS coated nanogenerators are measured and also taken the voltage across different resistances.

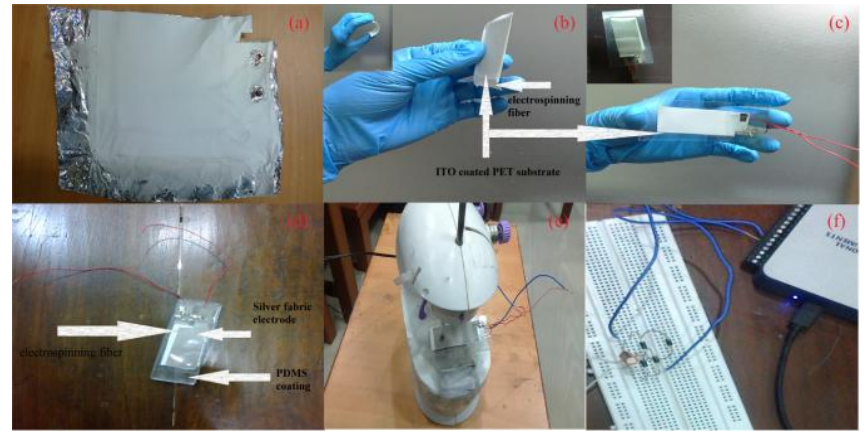

Fig.2. (a) Electrospinning fiber; (b) electrospinning fiber on ITO coated PET substrate.( inset shows its flexibility); (c) nanogenerator before PDMS coating.( inset shows reverse side of nanogenerator); (d) PDMS coated nanogenerator; (e) sewing machine for providing continuous thrust; (f) bridge circuit for capacitor charging. 


\section{Results And Discussion}

For characterization of nanofiber, ATR - FTIR , FE - SEM and UV - VIS instruments were used. We noticed and calculated different output voltage generation of these nanogenerator in different mechanical conditions. With the increase of resistance voltage drop across the resistance also increased. Then damping ratio of this nanogenerators has been calculated in cantilever geometry. After that we notice the output voltage in presence of light (green, blue) and mechanical stress (after PDMS coating). The capacitor charging voltage has also been found out of three nanogenerators respectively.

\subsection{FE - SEM analysis}

Figure 3 shows FE - SEM image of different AuPVDF doped electrospinning fiber and AuPVDF casted film. From fig 3(f), it can observe the presence of gold nanoparticle in AuPVDF film. Diameter of electrospinning nanofiber are varying from 100 - 1000 nanometer range.
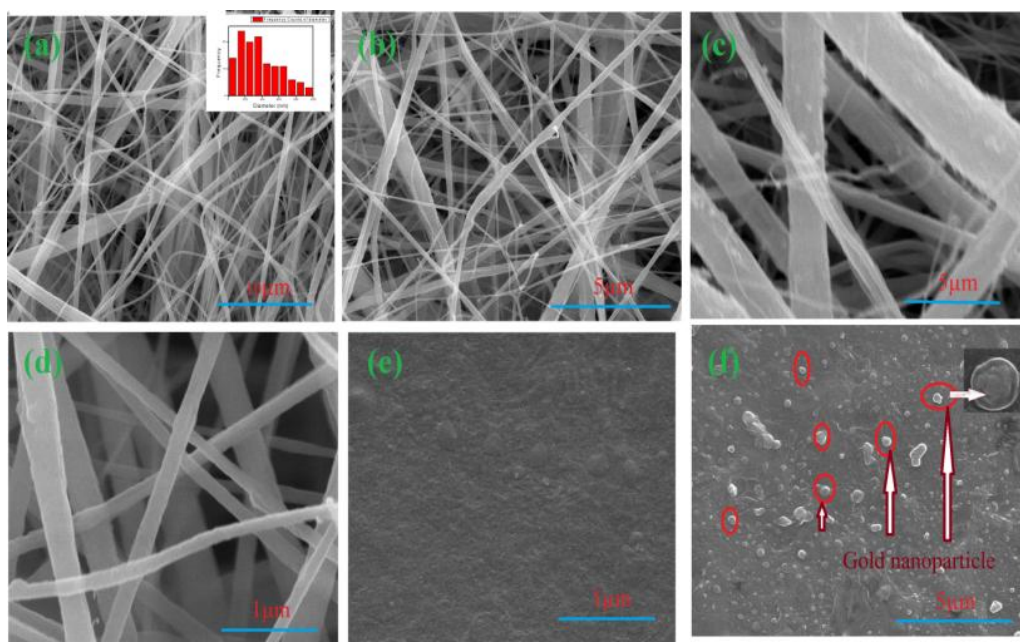

Fig.3. FE - SEM image of (a) Neat PVDF electrospinning fiber ( inset shows diameter distribution curve of nanofiber); (b) PVDF.700AuPVDF electrospinning fiber; (c) PVDF.500AuPVDF electrospinning fiber; (d) PVDF.300AuPVDF electrospinning fiber; (e) Neat PVDF film at $120^{\circ} \mathrm{c}$ (4 hrs. casting); (f) AuPVDF film at $120^{\circ} \mathrm{c}$ (4 hrs. casting).

\subsection{FTIR and temperature dependent FTIR analysis}

In case of electrospinning fiber[24,25,26,27] wavenumber $1275 \mathrm{~cm}^{-1}(\beta), 840 \mathrm{~cm}^{-1}(\beta)$ and $1234 \mathrm{~cm}^{-1} 1(\mathrm{\gamma})$ related absorbance peak is present but in case of casting film no $1275 \mathrm{~cm}^{-1}$ wavenumber related peak is present. The asymmetric and symmetric stretching vibrations of the $\mathrm{CH}_{2}$ group, respectively, at $3016 \mathrm{~cm}^{-1}$ and $2978 \mathrm{~cm}^{-}$ ${ }^{1}$ (Fig.5) are present in both electrospinning fiber and casting film. In case of all electrospinning fiber $2924 \mathrm{~cm}^{-1}$ wavenumber related peak is present but in case of casting film only PVDF.300AuPVDF casting film shows this kind of peak. In figure 4 and 5 different types of IR absorbance peak has been shown and corresponding wavenumber also.
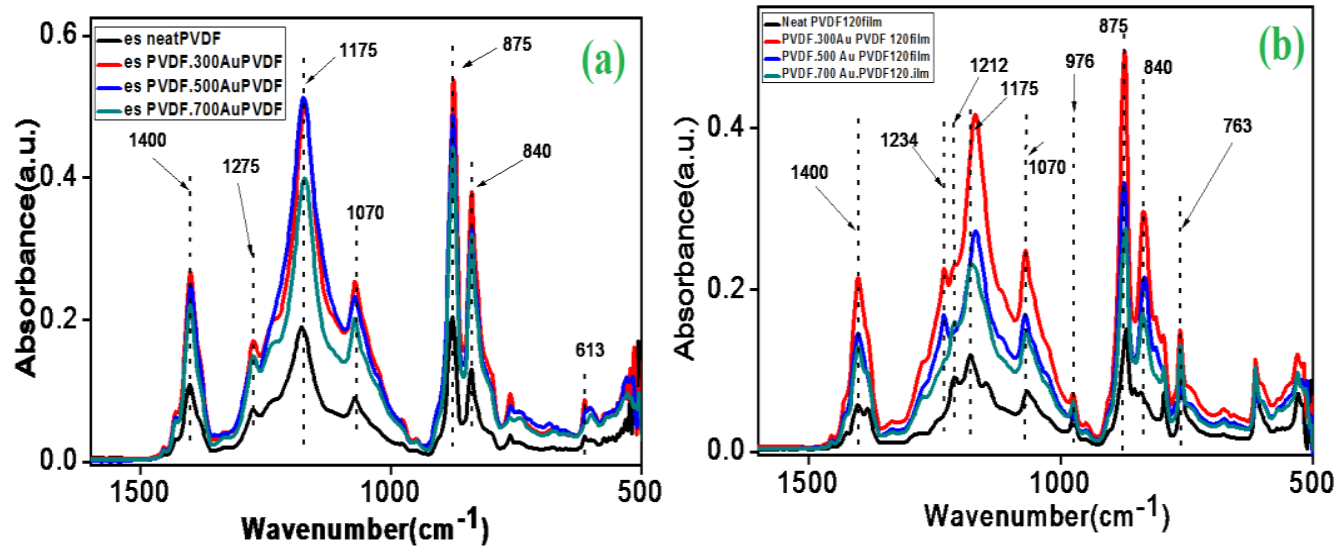

Fig.4. FTIR spectroscopy graph of (a) different electrospinning fiber; (b) casted films in $1600-500 \mathrm{~cm}^{-1}$ (wavenumber) range. 

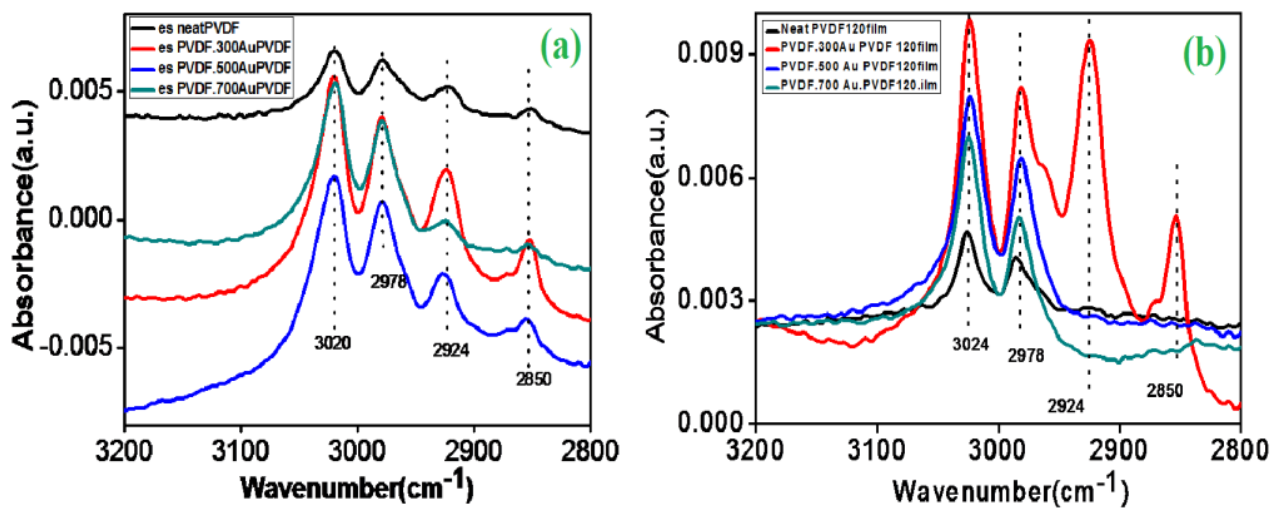

Fig.5. FTIR spectroscopy graph of (a) different electrospinning fiber; (b) casted film in $3200-2800 \mathrm{~cm}^{-1}$ (wavenumber) range.

From FTIR measurement of PVDF.500AuPVDF electrospinning fiber (Fig.6.(a)) at different temperature it is observe that at higher temperature $\left(175^{0} \mathrm{c}\right), \beta$ phase related peak $\left(1275 \mathrm{~cm}^{-1}\right)$ is vanished but $\gamma$ phase related peak $\left(838 \mathrm{~cm}^{-1}\right)$ is present. From this we can say that melting temperature of $\gamma$ phase is higher than $\beta$ phase. So $\gamma$ phase is thermally more stable than $\beta$ phase. From this figure it can also conclude that the IR absorbance at $1275 \mathrm{~cm}^{-1}$ wavenumber is higher than $838 \mathrm{~cm}^{-1}$ wavenumber.
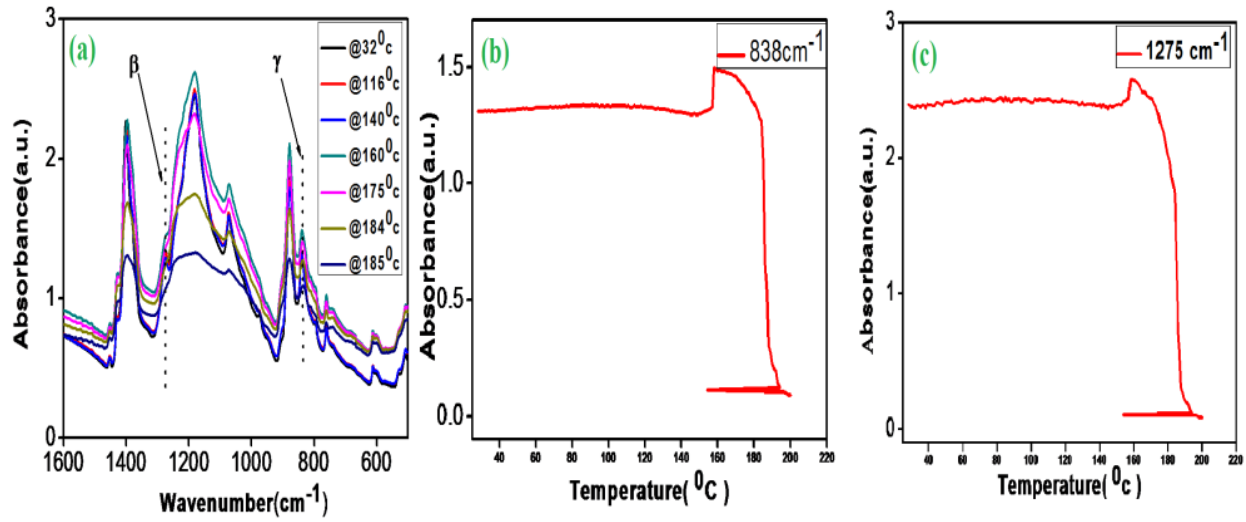

Fig.6. (a) Temperature dependent FTIR curve of PVDF.500AuPVDF electrospinning fiber; (b) absorbance vs temperature graph of $838 \mathrm{~cm}^{-1}$ wavenumber of PVDF.500AuPVDF electrospinning fiber; (c) absorbance vs temperature graph of $1275 \mathrm{~cm}^{-1}$ wavenumber of PVDF.500AuPVDF electrospinning fiber.

\subsection{UV - VIS analysis}

From UV - VIS curve it is observed a absorbance peak in between the wavelength of 500nm and $600 \mathrm{~nm}$ due to the surface plasmon resonance phenomena of gold nanoparticle which is present in PVDF solution. Fig. 7(b) shows AuPVDF solution and PVDF solution respectively. Colour change of AuPVDF solution is the primary evident of gold nanoparticle formation.

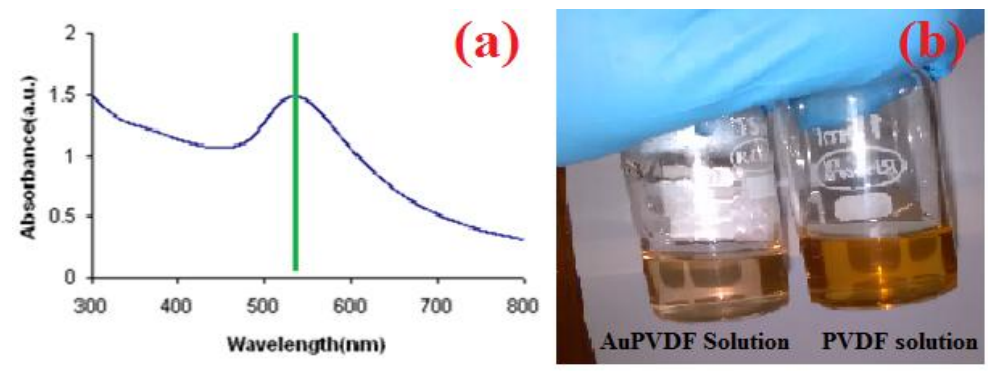

Fig.7. (a) UV - VIS spectroscopy curve of gold nanoparticle solution; (b) AuPVDF solution and PVDF solution. 
Gold Nanoparticle Doped PVDF Nanofiber Preparation Of Concurrently Harvesting Light...

\subsection{Electrical analysis}

The output voltage were also measured from our developed Ngs (PVDF.300AuPVDF, PVDF.500AuPVDF and PVDF.700AuPVDF) and PVDF.700AuPDVF nanogenerator gives maximum 100V (positive peak of AC output). It also shows maximum DC voltage of $\sim 25 \mathrm{~V}$ by heavily finger tapping .
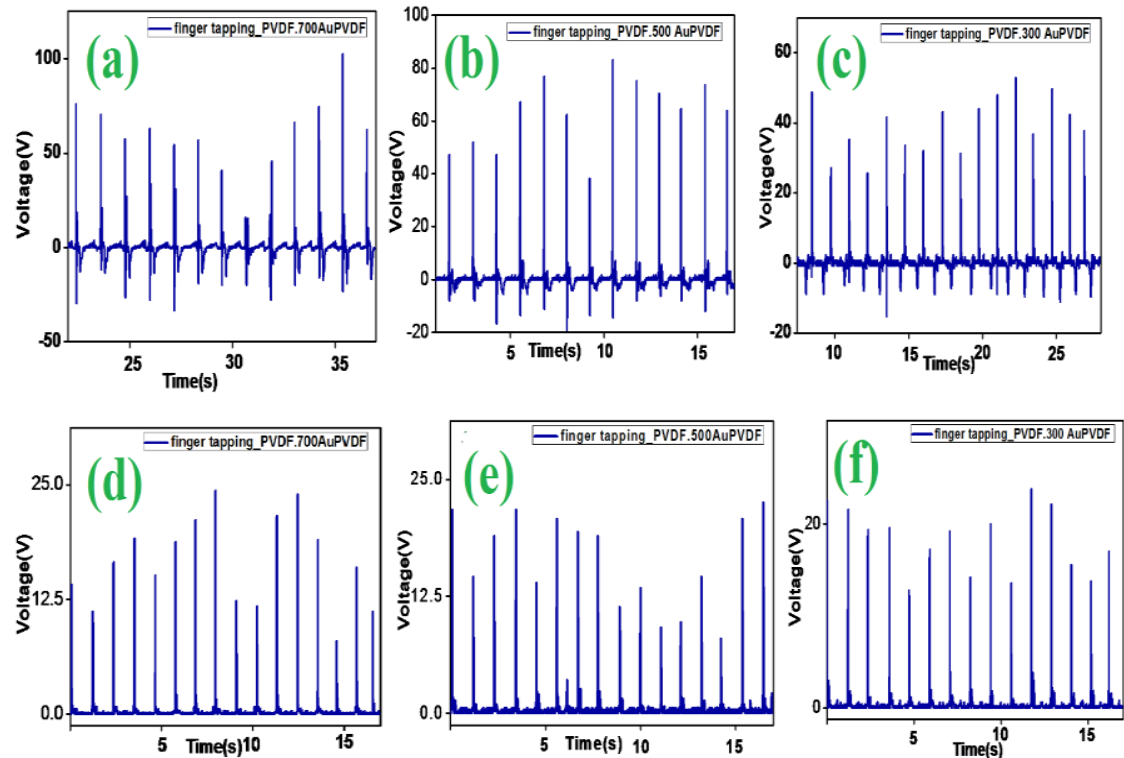

Fig.8. Output voltage from DSO by heavily finger tapping (a) AC output of PVDF.700AuPVDF Ng ; (b) AC output of PVDF.500AuPVDF Ng; (c) AC output of PVDF.300AuPVDF Ng; (d ) DC output of PVDF.700AuPVDF Ng; (e) DC output of PVDF.500AuPVDF Ng; (f) DC output of PVDF.300AuPVDF Ng.
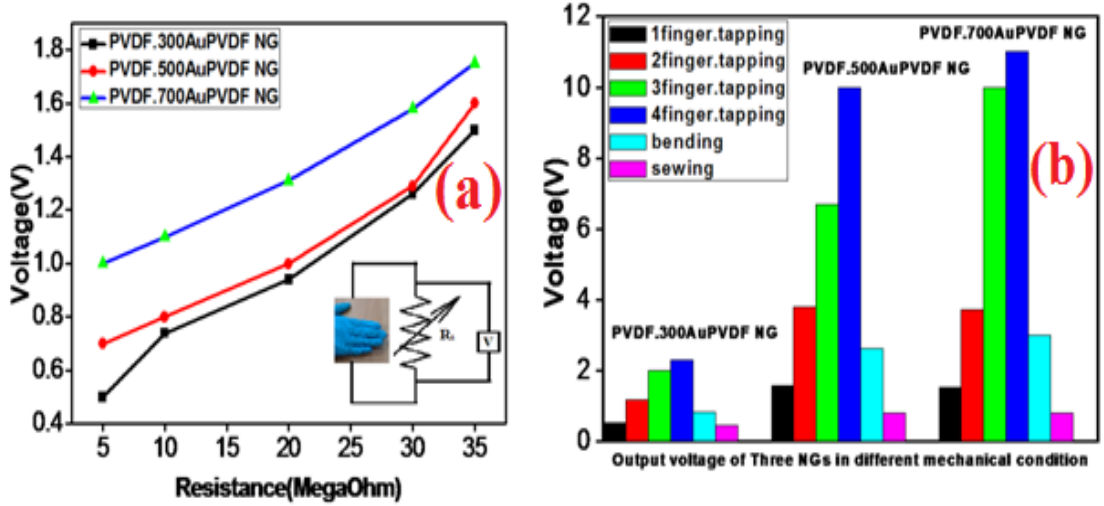

Fig.9. (a) Output Voltage across difference resistances (inset shows the circuit); (b) output of three nanogenerators in different mechanical condition.
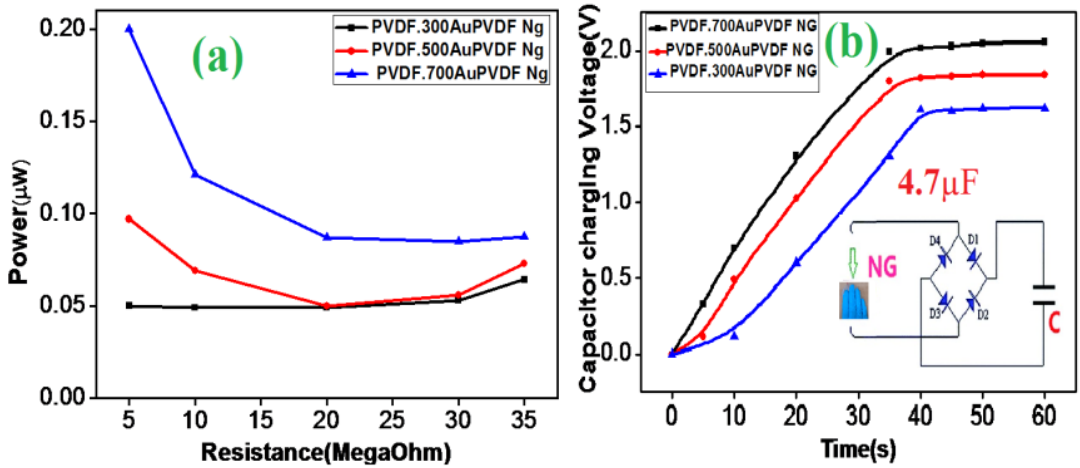

Fig.10. (a) Power consumption across different resistance; (b) capacitor charging voltage of three nanogenerators (inset shows charging circuit). 
Capacitor charging voltage across $4.7 \mu \mathrm{F}$ capacitor for these three nanogenerators respectively are $1.6 \mathrm{~V}, 1.8 \mathrm{~V}$, and 2.0V. (Capacitor charging circuit is shown in Fig2(f)). In cantilever geometry, before PDMS coating Damping ratio of PVDF.700AuPVDF nanognerator for thrust ( by one finger) given from $1 \mathrm{~cm}, 2 \mathrm{~cm}$, $3 \mathrm{~cm}, 4 \mathrm{~cm}$ distance respectively are $0.0084,0.011,0.01104,0.00488$, damping ratio of PVDF.500AuPVDF nanognerator for thrust given from $1 \mathrm{~cm}, 2 \mathrm{~cm}, 3 \mathrm{~cm}, 4 \mathrm{~cm}$ distance respectively are $0.0038,0.0036,0.033,0.0092$ and damping ratio of PVDF.300AuPVDF nanognerator for thrust given from $1 \mathrm{~cm}, 2 \mathrm{~cm}, 3 \mathrm{~cm}, 4 \mathrm{~cm}$ distance respectively are $0.0040,0.0059,0.0054,0.0063$.

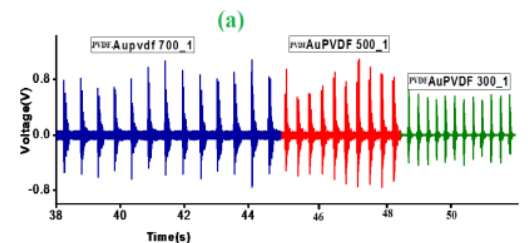

(c)

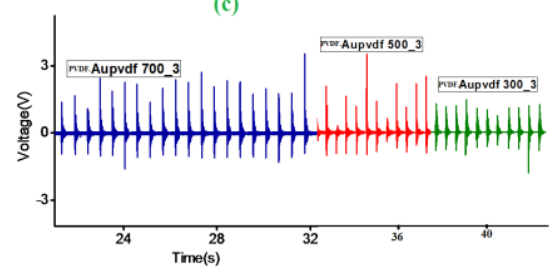

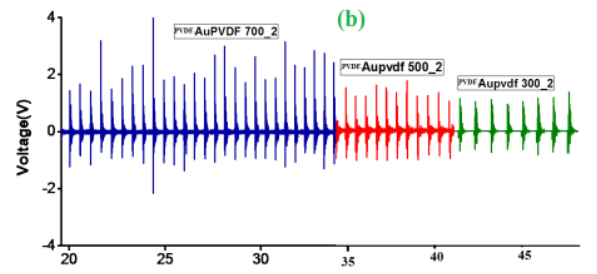

(d)

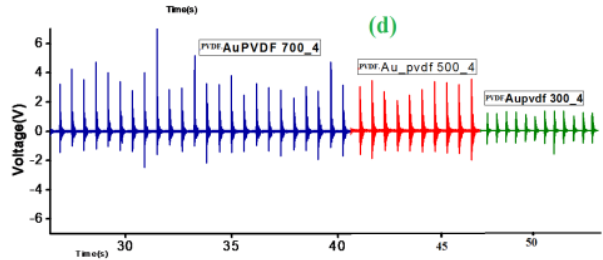

Fig.11. Output of three nanogenerators in cantilever geometry by finger thrust given by 1 finger from distance of (a) $1 \mathrm{~cm}$ away; (b) $2 \mathrm{~cm}$ away; (c) $3 \mathrm{~cm}$ away; (d) $4 \mathrm{~cm}$ away (blue response for PVDF.700AuPVDF nanogenerator, red for PVDF.500AuPVDF and green for PVDF.300AuPVDF nanogenerator).

From Fig. 11 it can observe that voltage is increasing for thrust given from $1 \mathrm{~cm}$ away to $4 \mathrm{~cm}$ away to this cantilever system and approximately in every cases PVDF.700AuPVDF nanogenerator gives maximum output voltage.

Then output voltages were measured in presence of sewing machine and blue/green light. From Fig.12 it is also observe that output voltage increases when blue light falls on these nanogenerators. but in case of green light(Fig.13) no such observation were noticed. So it can say gold nanoparticle doped nanogenerator is sensitive to blue light.

In future graphene oxide (GO) and/or reduced graphene oxide (RGO) can be incorporated into this electrospinning fiber for making better electrical and/or electronics properties so that it can able to generate more electrical voltage that means more power. Semiconductor quantum dots (QDs) also have been drawing great attention recently as a material for solar/light energy conversion due to their versatile optical and electrical properties. CdS, CdSe, $\mathrm{ZnS}$ quantum dot can also incorporate into this nanofiber for the improvement of efficiency of this kind of Nanogenerator where optical and mechanical energy are both concerned. It will be possible to transfer any data, charging our cell phone wirelessly.
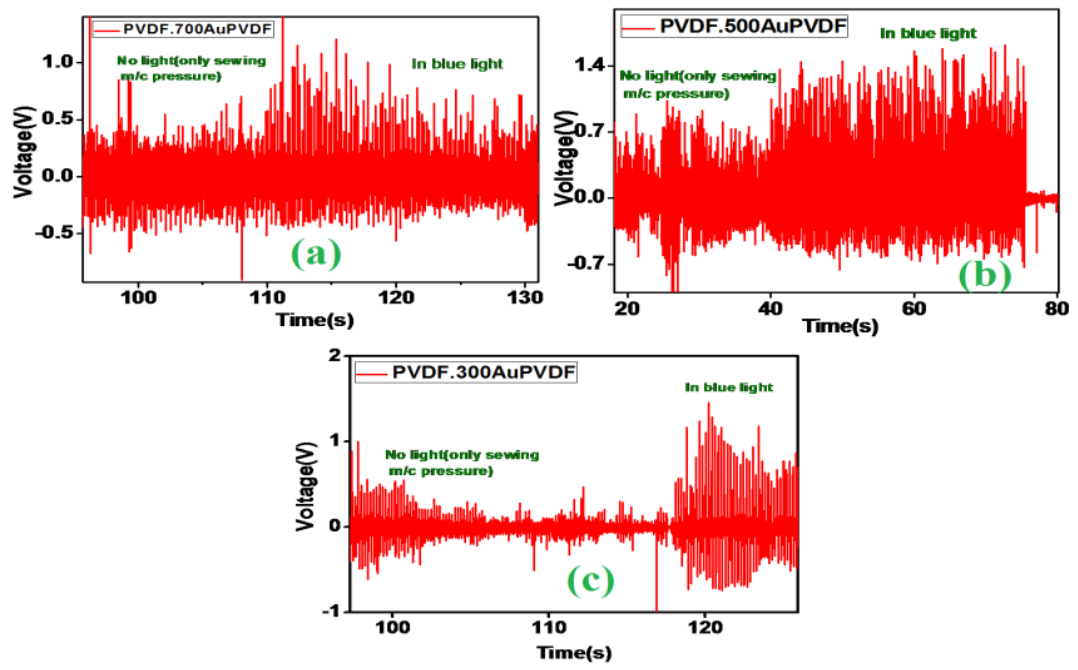

Fig.12. Output response of three nanognerators in no light condition (only sewing $\mathrm{m} / \mathrm{c}$ pressure) and in presence of both blue light and sewing machine pressure. 


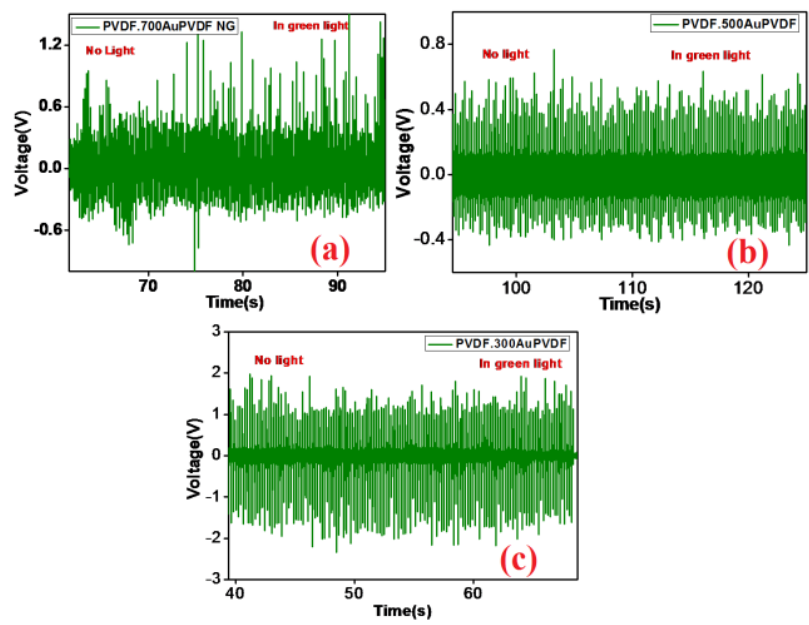

Fig.13. Output response of three nanognerators in no light condition (only sewing $\mathrm{m} / \mathrm{c}$ pressure) and in presence of both green light and sewing machine pressure.

\section{Acknowledgement}

Binoy Bera would like to thank Dr. Dipankar Mandal for giving him the opportunity to work in ONPDL laboratory. Binoy Bera performed most of the experiments. Author also want to thank Sujoy Ghosh of Department of physics, Jadavpur University for helping to prepare nanogenerator. Author also want to thank Kuntal Maity of Department of Physics, Jadavpur University for gold nanoparticle preparation. Binoy Bera wants to thank TEQIPphaseII,MAKAUT(Refno:09.02/Regis/Appnt.4/5) for providing him the fellowship.

\section{References}

[1]. Z. L. Wang, J. Song, Piezoelectric Nanogenerators Based on Zinc Oxide Nanowire Arrays, 2006 Science $312242-6$

[2]. C. Chang, V. H. Tran , J. Wang, Y. K. Fuh, L. Lin, Direct-write piezoelectric polymeric nanogenerator with high energy conversion efficiency, 2010 Nano Lett. $10726-31$.

[3]. Y. Qi, N.T. Jafferis, K. Lyons, C. M. Lee, H. Ahmad, M. C. McAlpine, Piezoelectric ribbons printed onto rubber for flexible energy conversion. 2010 Nano Lett. 10 524-8.

[4]. Y. Yang, L. Lin, Y. Zhang, Q. Jing, T. C. Hou, Z. L. Wang, Self-powered magnetic sensor based on a triboelectric nanogenerator, 2012 ACS Nano 6 10378-83.

[5]. S. B. Lang, 2005, Pyroelectricity: From Ancient Curiosity to Modern Imaging Tool, Phys. Today 58 31-6.

[6]. S. Xu, Y. Qin, C. Xu, Y. Wei, R. Yang, Z. L. Wang, Self-powered nanowire devices, 2010 Nature Nanotechnoly.5 $366-73$.

[7]. S. Bai, Q. Xu, L. Gu, F. Ma, Y. Qin, Z. L. Wang, single crystalline Lead Zirconate Titanae Nano/micro-wire based self-powered UV sensor, 2012 Nano Energy 1 789-95.

[8]. X. Y. Xue, . Wang S, W. Guo, Y. Zhang, Z. L. Wang, 2012 Nano Lett. Hybridizing energy conversion and storage in a mechanicalto-electrochemical process for self-charging power cell, 12 5048-54.

[9]. Y. Hu, Y. Zhang, C. Xu, G. Zhu, Z. L. Wang, 2010 Nano Lett. High-output nanogenerator by rational unipolar assembly of conical nanowires and its application for driving a small liquid crystal display, 10 5025-31.

[10]. S. Xu, B. J. Hansen, and Z. L. Wang, Piezoelectric-nanowire-enabled power source for driving wireless microelectronics, 2010 Nature Commun. 193

[11]. Z. L. Wang and J. H Song, "Piezoelectric nanogenerators based on zinc oxide nanowire arrays," Science, vol. 312, pp. 242-246, 2006.

[12]. M. Hussain, M. A. Abbasi, Z. H. Ibupoto, O. Nur and M. Willander, "The improved piezoelectric properties of ZnO nanorods with oxygen plasma treatment on the single layer graphene coated polymer substrate," Phys. Status. Soli. A, vol. 211, pp. 455-459, 2014

[13]. Z. T. Li, X. Zhang and G. H. Li, "In situ ZnO nanowire growth to promote the PVDF piezo phase and the ZnO-PVDF hybrid selfrectified nanogenerator as a touch sensor," Phys. Chem. Chem. Phys., vol. 16, pp. 5475-5479, 2014.

[14]. M. Y. Lu, J. Song, H. Lu, M. P. Lee, C. Y. Chen, L. J. and Z. L. Wang, "ZnO-ZnS Heterojunction and ZnS Nanowire Arrays for Electricity Generation," Acs Nano, vol. 3, pp. 357-362, 2009.

[15]. C. T. Huang, J. H. Song, W. F. Lee, Y. Ding, Z. Y. Gao, Y. Hao, L. J. Chen and Z. L. Wang, "GaN Nanowire Arrays for HighOutput Nanogenerators," J. Am. Chem. Soc., vol. 132, pp. 4766-4771, 2010.

[16]. J. Kwon, W. Seung, B. K. Sharma, S. W. Kim and J. H. Ahn, "A high performance PZT ribbon-based nanogenerator using graphene transparent electrodes," Energ. Environ. Sci., vol. 5, pp. 8970-8975, 2012

[17]. X. Chen, S. Y. Xu, N. Yao and Y. Shi, “1.6 V Nanogenerator for Mechanical Energy Harvesting Using PZT Nanofibers,” Nano. Lett., vol. 10, pp. 2133-2137, 2010.

[18]. Z. H. Lin, Y. Yang, J. M. Wu, Y. Liu, F. Zhang and Z. L. Wang, "BaTiO3 Nanotubes-Based Flexible and Transparent Nanogenerators," J. Phys. Chem. Lett., vol. 3, pp. 3599-3604, 2012.

[19]. P. Juan, X. J. Yan, Y. D. Jiang, C. Chang and L. W. Lin, "Piezoelectric actuation of direct-write electrospun fibers," Sensor. Actuat. A- Phys., vol.164, pp. 131-136, 2010.

[20]. Binoy Bera, Madhumita.Das Sarkar, "Piezoelectricity in PVDF and PVDF Based Piezoelectric Nanogenerator: A Concept”. IOSR Journal of Applied Physics (IOSR-JAP). Volume 9, Issue 3 Ver. I, PP 95-99.

[21]. Z. H. Liu, C. T. Pan, L. W. Lin and H. W. Lai, "Piezoelectric properties of PVDF/MWCNT nanofiber using near-field electrospinning," Sensor.Actuat. A-Phys., vol. 193, pp. 13-24, 2013. 
[22]. H. Yu, T. Huang, M. X. Lu, M. Y. Mao, Q. H. Zhang and H. Z. Wang, "Enhanced power output of an electrospun PVDF/MWCNTs-based nanogenerator by tuning its conductivity," Nanotechnology," vol. 24, pp. 405401 (9 pp.)-405401 (9 pp.), 2013.

[23]. X. Huang, P.K. Jain, I.H. El-Sayed, M.A. El-Sayed, "Gold nanoparticles: interesting optical properties and recent applications in cancer diagnostics and therapy,” Nanomed, 2 (5) (2007), pp. 681-693.

[24]. Binoy Bera, Madhumita.Das Sarkar, "PVDF based Piezoelectric Nanogenerator as a new kind of device for generating power from renewable resources". IOSR Journal of Polymer and Textile Engineering (IOSR-JPTE). Volume 4, Issue 2 (Mar. - Apr. 2017), PP 01-05.

[25]. B. Bera, Literature Review on Electrospinning Process (A Fascinating Fiber Fabrication Technique), Imperial Journal of Interdisciplinary Research (IJIR) Vol-2, Issue-8, 2016.

[26]. Binoy Bera, Dipankar Mandal, Madhumita Das Sarkar, Sensor Made of PVDF/graphene Electrospinning Fiber and Comparison between Electrospinning PVDF Fiber and PVDF/graphene FiberImperial Journal of Interdisciplinary Research (IJIR) Vol-2, Issue-5, 2016

[27]. D. R. Dillon, K. K. Tenneti, C. Y. Li, F. K. Ko, I. Sics and B. S. Hsiao, "On the structure and morphology of polyvinylidene fluoride- nanoclay nanocomposites," Polymer, vol. 47, pp. 1678-1688, 2006. 